Original Research Paper

\title{
Robust and Efficient Controller to Design a Standalone Source Supplied DC and AC Load Powered by Photovoltaic Generator
}

\author{
Hassan Abouobaida \\ Laboratory of Engineering Sciences for Energy, \\ National School of Applied Sciences of El Jadida, Chouaib-Doukkali University, Morocco
}

Article history

Received: 29-10-2014

Revised: 02-09-2016

Accepted: 25-10-2016

Email: hassanabouobaida@gmail.com

\begin{abstract}
The present work describes the analysis, modeling and control of a cascade DC-DC and DC-AC power conditioning stage to control a output voltage to supply a DC and AC load systems. To maximize energy extracted from PV generator and control output voltage a RCC MPPT and backstepping controller are designed. The stability of the control algorithm is demonstrated by means of Lyapunov analysis. The achievement of the DC-DC and DC-AC conversions and the efficient PV's energy extraction are validated with simulation results.
\end{abstract}

Keywords: MPPT, Backstepping Controller, DC-DC Converter, DC-AC Inverter

\section{Introduction}

Photovoltaic (PV) is a technology in which the radiant energy from the sun is converted to direct current. The photovoltaic process produces power silently and is completely self-contained, as there are no moving parts. These systems can withstand severe weather conditions including snow and ice. Photovoltaic systems for different applications can be either stand alone or grid connected. In a standalone system, the load has no connection to the utility grid and often relies on a set of batteries to secure an energy supply at night and other times when the solar panels do not produce electricity. A utility interactive or grid connected system is employed in applications where utility service is already available. In this case, there is no need for battery storage because the power station can be used to supplement photovoltaic generation when the load exceeds the available PV output. The use of photovoltaic as the power source with regulated voltage (DC $12 \mathrm{~V}-24$ or AC $220 \mathrm{~V}$ ) is considered as one of the most important application of PV system. Photovoltaic powered DC system allows to adapt the output voltage to the load even for climatic conditions that may change.

A power conditioning system linking the solar array and the power DC or AC load is needed to facilitate an efficient energy transfer between them, this implies that the power stage has to be able to extract the maximum amount of energy from the PV and to control à voltage of DC load.
In order to extract the maximum amount of energy the PV system must be capable of tracking the solar arrays Maximum Power Point (MPP) that varies with the solar radiation value and temperature. Several MPPT algorithms have been proposed, namely, Perturb and Observe (P\&O), incremental conductance, RCC algorithms (Casadei et al., 2006; Liu et al., 2013), etc. They differ from its complexity and tracking accuracy but they all required sensing the PV current and/or the PV voltage.

Several controller strategies have been used in the literature, citing the PID that is generally suitable for linear systems, the sliding mode for which the chattering problem and fuzzy logic adapted to systems without a mathematical model (Lalouni and Rekioua, 2009).

In this study, the problem of controlling switched power converters is approached using the backstepping technique. While feedback linearization methods require precise models and often cancel some useful nonlinearities, backstepping designs offer a choice of design tools for accommodation of uncertain nonlinearities and can avoid wasteful cancellations. The backstepping approach is applied to a specific class of switched power converters, namely dc-to-dc converters. In the case where the converter model is fully known the backstepping nonlinear controller is shown to achieve the control objectives i.e., input voltage tracking and voltage control of $\mathrm{DC}$ or $\mathrm{AC}$ load with respect to climate 
change. The desired array voltage is designed online using a Ripple Correlation Current (RCC) MPP tracking algorithm (Barth and Pilawa-Podgurski, 2013). The proposed strategy ensures that the MPP is determined, the voltage of DC or AC load is controlled to its reference value and the close loop system will be asymptotically stable. The stability of the control algorithm is analysed by Lyapunov approach. The rest of the paper is organized as follows. The dynamic model of the global system is described in Section II. A backstepping controller is designed along with the corresponding closed-loop error system and the stability analysis is discussed in Section III. In Section IV, a simulation results proves the effectiveness of this approach with constant solar radiation and temperature.

\section{MPPT System Modelling}

The solar generation model consists of a PV array module, dc-to-dc boost converter, dc-to-dc buck converter as shown in Fig. 1.

\section{PV Model}

PV array is a p-n junction semiconductor, which converts light into electricity. When the incoming solar energy exceeds the band-gap energy of the module, photons are absorbed by materials to generate electricity. The equivalent-circuit model of PV is shown in Fig. 2. It consists of a light-generated source, diode, series and parallel resistances (Rekioua and Matagne, 2012).

\section{Boost Converter Model}

The dynamic model of the power boost converter presented in Fig. 3 can be expressed by an instantaneous switched model as follows Equation 1 and 2:

$$
\begin{aligned}
& c_{1} \cdot \dot{u}_{p v}=I_{p v}-I_{L_{1}} \\
& L_{1} \cdot I_{L_{1}}=u_{p v}-\left(1-u_{1}\right) \cdot u_{d c}
\end{aligned}
$$

where, $L_{1}$ and $I_{L 1}$ represents the dc-to-dc converter storage inductance and the current across it, $u_{d c}$ is the output voltage and $u_{1}$ is the switched control signal.

Using the state averaging method, the switched model can be redefined by the average PWM model as follows:

$$
\begin{aligned}
& c_{1} \dot{\bar{u}}_{p v}=\bar{I}_{p v}-\bar{I}_{L 1} \\
& L \cdot \dot{\bar{I}}_{L}=\bar{u}_{p v}-\alpha_{1} \cdot \bar{u}_{d c}
\end{aligned}
$$

where, $\alpha_{1}$ is averaging value of $\left(1-u_{1}\right), \bar{u}_{p v}$ and $\bar{I}_{p v}$ are the average states of the output voltage and current of the PV generator, $\bar{I}_{L 1}$ is the average state of the inductor current.

\section{Buck Converter Model}

The power converter here is like buck. It provides power to the DC load with a share of power supplied by the photovoltaic generator and the battery source. The main control objective of the buck converter is to accomplish constant voltage operation of the DC load. Figure 4 illustrate a buck converter connected to load.

Noticing that $\mathrm{u}_{2}$ stand for the control signal of buck converter, the system can be represented by differential Equation 5 and 6:

$c_{2} \cdot \dot{u}_{s 1}=I_{L 2}-\frac{u_{s 1}}{R_{1}}$

$L_{2} \cdot \dot{I}_{L 2}=u_{2} \cdot u_{d c}-u_{s 1}$

where, $u_{d c}$ and $u_{s}$ designs a battery DC voltage and output buck converter voltage respectively.

$L_{2}, I_{L 2}$ and $R_{L}$ are the dc-to-dc converter storage inductance, the current across it and load resistor. $u_{2}$ is the switched control signal.

Using the state averaging method, the switched buck converter model can be redefined by the average PWM model as follows:

$c_{2} \cdot \dot{\bar{u}}_{s 1}=\bar{I}_{L 2}-\frac{\bar{u}_{s 1}}{R_{1}}$

$L_{2} \cdot \dot{\bar{I}}_{L 2}=\alpha_{2} \cdot \bar{u}_{d c}-\bar{u}_{s 1}$

where, $\alpha_{2}$ is averaging value of $u_{2}$ and $\bar{u}_{s}$ is the average states of the output.

\section{Buck Inverter Model}

The active power transfer from the PV panels is accomplished by regulating the voltage that supply the single phase AC load. The inverter operates as a Voltage-Control Inverter (VCI). Figure 5 illustrate a buck inverter connected to load.

Noticing that $u_{3}$ stand for the control signal of the inverter, the system can be represented by differential Equation 9 and 10:

$$
\begin{aligned}
& c_{3} \cdot \dot{u}_{s 2}=i_{L 3}-i_{R 2} \\
& L_{3} \cdot \dot{i}_{L 3}=\left(2 \cdot u_{3}-1\right) \cdot u_{d c}-u_{s 2}
\end{aligned}
$$

where, $u_{d c}, u_{s 2}$ designs a battery voltage and load voltage respectively. $i_{L 3}, i_{L 2}$ are inverter output current and load current respectively. 


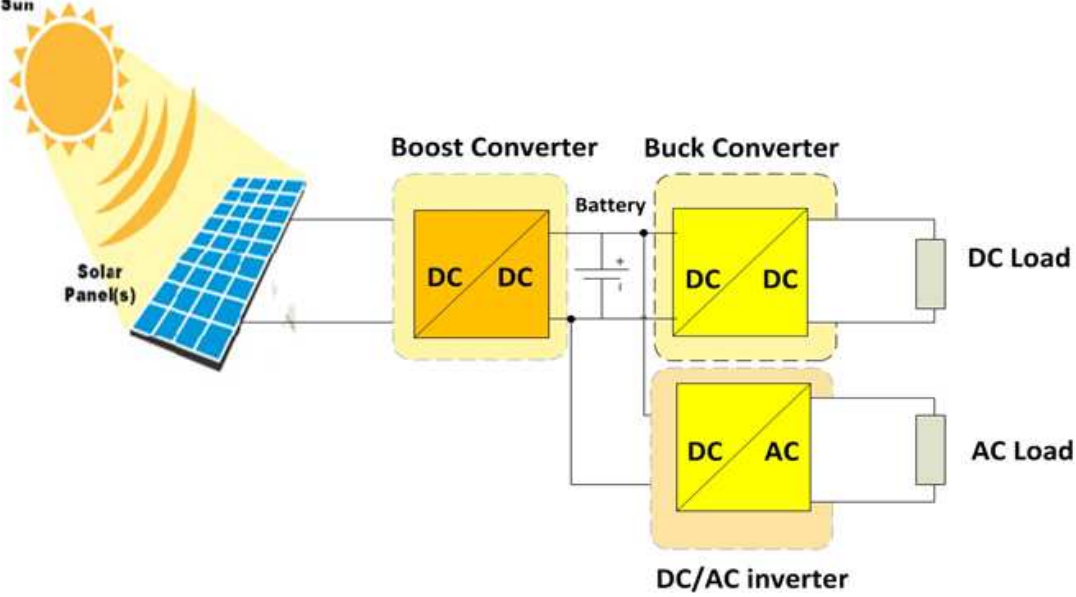

Fig. 1. Studied PV system

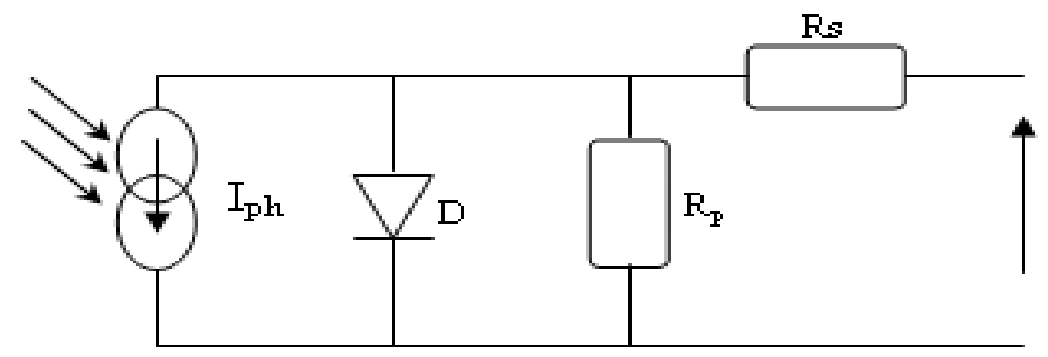

Fig. 2. Equivalent model of PV cell

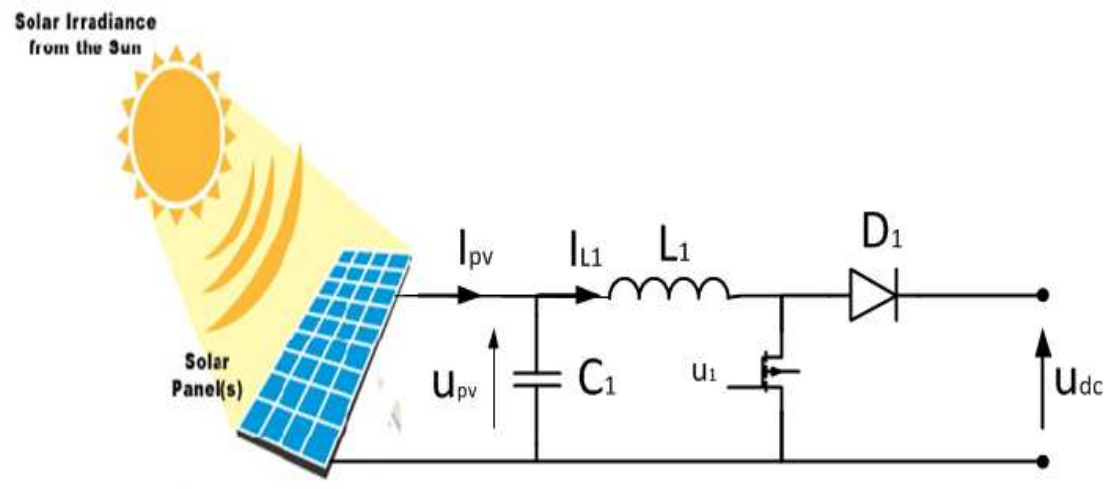

Fig. 3. PV generator and Boost converter

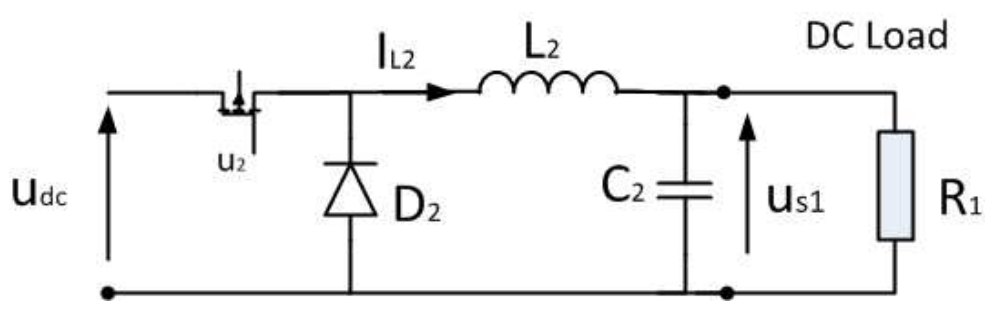

Fig. 4. Buck converter and DC load 


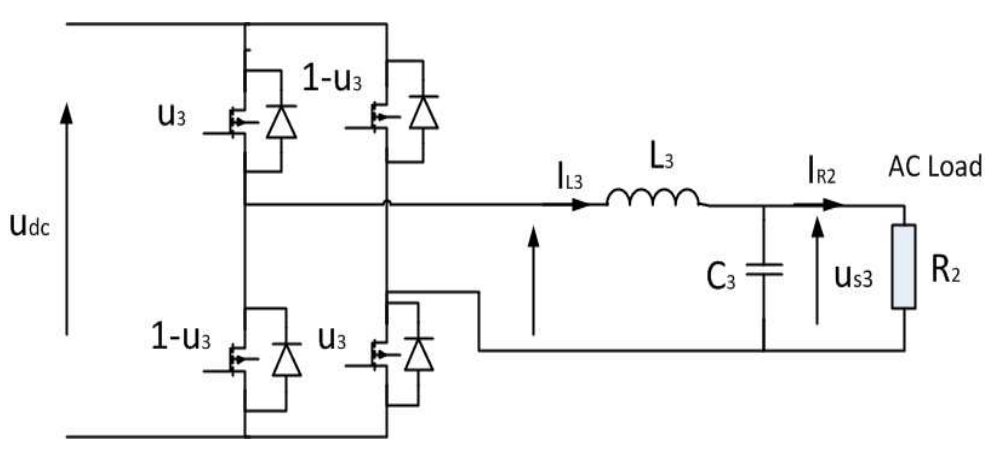

Fig. 5. Buck inverter

Using the state averaging method (on cutting period), the switched model can be redefined by the average PWM model as follows :

$$
\begin{aligned}
& c_{3} \cdot \dot{\bar{u}}_{s 2}=\bar{i}_{L 3}-\bar{i}_{R 2} \\
& L_{3} \dot{\bar{i}}_{L 3}=\left(2 \cdot \alpha_{3}-1\right) \cdot \bar{u}_{d c}-\bar{u}_{s 2}
\end{aligned}
$$

where, $\alpha_{3}$ is averaging value of $u_{3}$

\section{Nonlinear Controller Design}

Two main objectives have to be fulfilled in order to transfer efficiently the photovoltaic generated energy into the DC and/or AC load are tracking the PV's Maximum Power Point (MPP) and control output voltage of DC and AC load. Figure 6 shows the control scheme used to accomplish the previous objectives.

\section{Backstepping Controller to Extract Maximum Power}

The boost converter is governed by control signal $\alpha_{1}$ generated by a backstepping controller that allow to extract maximum of photovoltaic generator control by regulating the voltage of the photovoltaic generator to its reference provided by MPPT algorithm.

Step 1. Let us introduce the input error Equation 13:

$$
e_{1}=\bar{u}_{p v}-u_{p v} *
$$

Deriving $e_{1}$ with respect to time and accounting for (3), implies Equation 14:

$$
\dot{e}_{1}=\dot{\bar{u}}_{p v}-\dot{u}_{p v} *\left(\frac{\bar{I}_{p v}}{c_{1}}-\frac{\bar{I}_{L_{1}}}{c_{1}}\right)-\dot{u}_{p v} *
$$

In Equation 14, $i_{L 1}$ behaves as a virtual control input. Such an equation shows that one gets $\dot{e}_{1}=-k_{1} \cdot e_{1}\left(k_{1}>0\right.$ being a design parameter) provided that:

$$
I_{L_{1}}=k_{1} \cdot c_{1} \cdot e_{1}+\bar{I}_{p v}-c_{1} \cdot \dot{u}_{p v} *
$$

As $i_{L 1}$ is just a variable and not (an effective) control input, (14) cannot be enforced for all $t \geq 0$. Nevertheless, Equation 12 shows that the desired value for the variable $\mathrm{I}_{\mathrm{L} 1}$ is Equation 16:

$I_{L_{1}}{ }^{*}=k_{1} \cdot c_{1} \cdot e_{1}+I_{p v}-c_{1} \cdot \dot{u}_{p v} *$

Indeed, if the error Equation 17:

$e_{2}=I_{L_{1}}-I_{L_{1}} *$

Vanishes (asymptotically) then control objective is achieved i.e., $e_{1}=u_{p v}-u_{p v}{ }^{*}$ vanishes in turn. The desired value $I_{L 1} *$ is called a stabilization function.

Now, replacing $i_{L 1}$ by $\left(e_{2}+I_{L 1}{ }^{*}\right)$ in (14) yields Equation 18:

$\dot{e}_{1}=\left(\frac{\bar{I}_{p v}}{c_{1}}-\frac{\bar{I}_{L_{1}} *+e_{2}}{c_{1}}\right)-\dot{u}_{p v} *$

Which, together with (16), gives Equation 19:

$\dot{e}_{1}=-k_{1} \cdot e_{1}-\frac{e_{2}}{c_{1}}$

Step 2. Let us investigate the behavior of error variable $e_{2}$.

In view of (4) and (17), time-derivation of $e_{2}$ turns out to be Equation 20:

$\dot{e}_{2}=\dot{\bar{I}}_{L_{1}}-\dot{I}_{L_{1}} *=\left(\frac{\bar{u}_{\mathrm{pv}}}{L_{1}}-\frac{\alpha_{1} \cdot \bar{u}_{d c}}{L_{1}}\right)-\dot{I}_{L_{1}} *$

From (16) one gets Equation 21:

$\dot{I}_{L_{1}}{ }^{*}=k_{1} \cdot c_{1} \cdot \dot{e}_{1}+\dot{\bar{I}}_{p v}-c_{1} \cdot \ddot{u}_{p v} *$ 


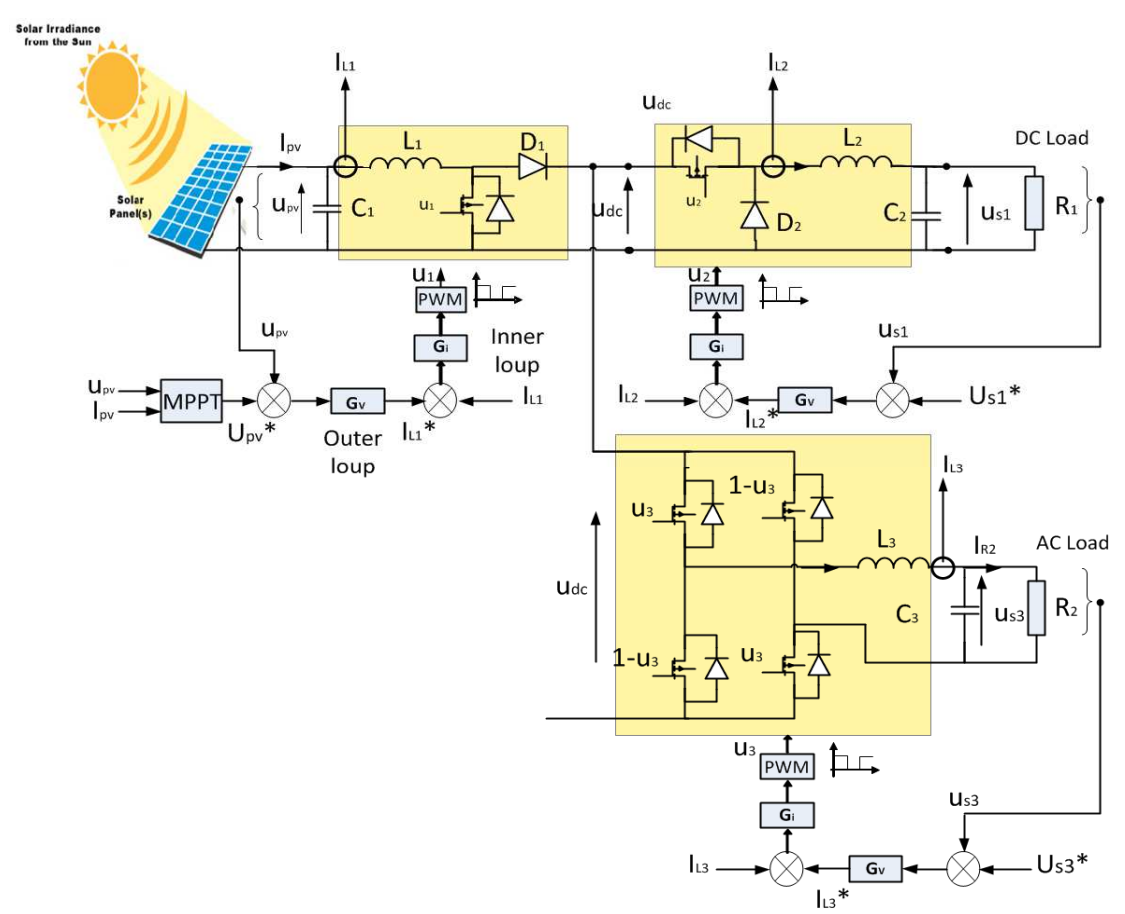

Fig. 6. Control strategy

Which together with (20) implies Equation 22:

$\dot{e}_{2}=\frac{\bar{u}_{\mathrm{pv}}}{L_{1}}-\frac{\alpha_{1} \cdot \bar{u}_{d c}}{L_{1}}-k_{1} \cdot c_{1} \cdot \dot{e}_{1}-\dot{\bar{I}}_{p v}+c_{1} \cdot \ddot{u}_{p v} *$

\section{Buck Controller Design}

This controller consists of an inner current loop and an outer voltage loop. The inner current loop is responsible to regulate the current of storage inductance in buck converter. The outer loop assures a steady-state control a output voltage.

Step 1. Let us introduce the input error Equation 23:

$e_{3}=\bar{u}_{s 1}-u_{s 1} *$

where, $u_{s 1}{ }^{*}$ is a derivable reference signal of output voltage of DC load. Deriving $e_{3}$ with respect to time and accounting for (7) implies Equation 24:

$\dot{e}_{3}=\dot{\bar{u}}_{s 1}-\dot{u}_{s 1}^{*}=\frac{\bar{I}_{L 2}}{c_{2}}-\frac{\bar{u}_{s 1}}{R_{2} \cdot c_{2}}$

where, $I_{L 2}$ is a virtual control input. Such an equation shows that one gets $\dot{e}_{3}=-k_{3} \cdot e_{3} \quad\left(k_{3}>0\right.$ being a design parameter) provided that:

$I_{L 2} *=\frac{\bar{u}_{s 1}}{R_{1}}-k_{3} \cdot e_{3} \cdot c_{2}$
As $I_{L 2} *$ is just a variable (not effective) control input, Equation 25 cannot be enforced for all $t \geq 0$. Indeed, a new error is introduced Equation 26:

$e_{4}=\bar{I}_{L 2}-I_{L 2}{ }^{*}=\bar{I}_{L 2}-\left[\frac{\bar{u}_{s 1}}{R_{1}}-k_{3} \cdot e_{3} \cdot c_{2}\right]$

Vanishes (asymptotically) then control objective is achieved i.e., $e_{3}=\bar{u}_{s 1}-u_{s 1} *$ vanishes in turn. The desired value $I_{L 2}{ }^{*}$ is called a stabilization function.

Now, replacing $\mathrm{I}_{\mathrm{L} 2}$ by $\left(i_{L 2} *+e_{4}\right)$ in (24) yields Equation 27:

$\dot{e}_{3}=\frac{I_{L 2} *+\mathrm{e}_{4}}{c_{2}}-\frac{u_{s 1}}{R_{1} \cdot c_{2}}$

Which, together with (25), gives Equation 28:

$\dot{e}_{3}=-k_{3} \cdot e_{3}+\frac{e_{4}}{c_{2}}$

Step 2. Let us investigate the behavior of error variable $e_{4}$.

In view of (8), time-derivation of $e_{4}$ turns out to be Equation 29:

$\dot{e}_{4}=\dot{I}_{L 2}-\dot{I}_{L 2} *=\left(\frac{\alpha_{2} \cdot \bar{u}_{d c}}{L_{2}}-\frac{\bar{u}_{s 1}}{L_{2}}\right)-\dot{I}_{L 2} *$

From (25) one gets Equation 30: 


$$
\dot{I}_{L 2} *=\frac{\dot{\bar{u}}_{s 1}}{R_{1}}-k_{3} \cdot \dot{e}_{3} \cdot c_{2}
$$

Substituting (30) in (29) implies Equation 31:

$$
\dot{e}_{4}=\frac{\alpha_{2} \cdot \bar{u}_{d c}}{L_{2}}-\frac{\bar{u}_{s 1}}{L_{2}}-\left(\frac{\dot{\bar{u}}_{s 1}}{R_{1}}-k_{3} \cdot \dot{e}_{3} \cdot c_{2}\right)
$$

\section{Control Design of VCI Inverter}

This controller consists of an inner current loop and an outer voltage loop. The inner current loop is responsible of producing the averaging value of switched control signal. The outer voltage loop assures a steady-state maximum input-output energy transfer ratio and regulate a desired steady-state output voltage that supply the AC load.

Step 1. Let us introduce the input error Equation 32:

$e_{5}=u_{s 2}-u_{s 2} *$

where, $u_{s 2} *$ is a reference signal of the output voltage.

Deriving $e_{5}$ with respect to time and accounting for (11) implies:

$\dot{e}_{5}=\dot{\bar{u}}_{s 2}-\dot{\bar{u}}_{s 2} *=\frac{\bar{i}_{L 3}-\bar{i}_{R 2}}{c_{3}}-\dot{\bar{u}}_{s 2} *$

In Equation 33, $\bar{i}_{L 3}$ behaves as a virtual control input. Such an equation shows that one gets $\dot{\mathrm{e}}_{5}=-\mathrm{k}_{5} \cdot \mathrm{e}_{5}\left(\mathrm{k}_{5}>0\right.$ being a design parameter) provided that:

$\bar{i}_{L 3}=-c_{3} \cdot k_{5} \cdot e_{5}+c_{3} \cdot \dot{\bar{u}}_{s 2} *+i_{r 2}$

As $\bar{i}_{L 3}$ is just a variable and not (an effective) control input, (34) cannot be enforced for all $t \geq 0$. Nevertheless, Equation 34 shows that the desired value for the variable $\bar{i}_{L 3}$ is Equation 25:

$\bar{i}_{L 3} *=-c_{3} \cdot k_{5} \cdot e_{5}+c_{3} \cdot \dot{\bar{u}}_{s 2} *+i_{r 2}$

Indeed, if the error Equation 36:

$e_{6}=\bar{i}_{L 3}-\bar{i}_{L 3} *$

Now, replacing $\mathrm{i}_{\mathrm{L} 3}$ by $\left(\mathrm{e}_{6}+\mathrm{I}_{\mathrm{L} 3} *\right)$ in (33) yields Equation 37:

$\dot{e}_{5}=\frac{\left(e_{6}+i_{L 3}{ }^{*}\right)-\bar{i}_{R 2}}{c_{3}}-\dot{\bar{u}}_{s 2} *$

Which, together with (35), gives Equation 38: $\dot{e}_{5}=-k_{5} \cdot e_{5}+\frac{e_{6}}{c_{3}}$

Step 2. Let us investigate the behavior of error variable $e_{4}$.

In view of (12), time-derivation of $e_{6}$ turns out to be Equation 39:

$\dot{e}_{6}=\dot{\bar{i}}_{L 3}-\dot{\bar{i}}_{L 3} *=\frac{\left(2 \cdot \alpha_{3}-1\right) \bar{u}_{d c}-\bar{u}_{s 2}}{L_{3}}-\dot{\bar{i}}_{L 3} *$

Which together with (26) implies Equation 40:

$\dot{e}_{6}=\frac{\left(2 \cdot \alpha_{3}-1\right) \cdot \bar{u}_{d c}-\bar{u}_{s 2}}{L_{3}}+c_{3} \cdot k_{5} \cdot \dot{e}_{5}-c_{3} \cdot \ddot{\bar{u}}_{s 2} *-\dot{\overline{i_{r 2}}}$

In the new coordinates $\left(e_{1}, e_{2}, e_{3}, e_{4}, e_{5}, e_{6}\right)$, the controlled system is expressed by the couple of Equation $3,4,7,8,11$ and 12. We now need to select a Lyapunov function for such a system. As the objective is to drive its states $\left(e_{1}, e_{2}, e_{3}, e_{4}, e_{5}, e_{6}\right)$ to zero, it is natural to choose the following function Equation 41:

$V_{2}=\frac{1}{2} \cdot e_{1}^{2}+\frac{1}{2} \cdot e_{2}^{2}+\frac{1}{2} \cdot e_{3}^{2}+\frac{1}{2} \cdot e_{4}^{2}+\frac{1}{2} \cdot e_{5}^{2}+\frac{1}{2} \cdot e_{6}^{2}$

The time-derivative of the latter, along the $\left(e_{1}, e_{2}, e_{3}, e_{4}, e_{5}, e_{6}\right)$ trajectory is Equation 42 and 43:

$\dot{V}_{2}=e_{1} \dot{e}_{1}+e_{2} \cdot \dot{e}_{2}+e_{3} \cdot \dot{e}_{3}+e_{4} \cdot \dot{e}_{4}+e_{5} \cdot \dot{e}_{5}+e_{6} \cdot \dot{e}_{6}$

$\dot{V}_{2}=-k_{1} \cdot e_{1}^{2}-k_{2} \cdot e_{2}^{2}-k_{3} \cdot e_{3}^{2}-k_{4} \cdot e_{4}^{2}-k_{5} \cdot e_{5}^{2}-k_{6} \cdot e_{6}^{2}$

The equilibrium $\left(e_{1}, e_{2}, e_{3}, e_{4}, e_{2} e_{2}\right)=(0,0)$ is globally asymptotically stable, so doing, one gets the following control law Equation 44 to 46:

$\alpha_{1}=\frac{L_{1}}{\bar{u}_{d c}}\left[\frac{\bar{u}_{\mathrm{pv}}}{L_{1}}-\frac{e_{1}}{c_{1}}+k_{2} \cdot e_{2}-k_{1} \cdot c_{1} \cdot \dot{e}_{1}-\dot{\bar{I}}_{p v}+c_{1} \cdot \ddot{u}_{p v} *\right]$

$\alpha_{2}=\frac{L_{2}}{\bar{u}_{d c}} \cdot\left[\frac{\bar{u}_{s 1}}{L_{2}}-\frac{\dot{\bar{u}}_{s 1}}{R_{1}}-k_{3} \cdot \dot{e}_{3} \cdot c_{2}-\frac{e_{3}}{c_{2}}-k_{4} \cdot e_{4}\right]$

$\alpha_{3}=\frac{1}{2}+\frac{L_{3}}{\bar{u}_{d c}}\left[\frac{e_{5}}{c_{3}}+\frac{\bar{u}_{s 2}}{L_{3}}+c_{3} \cdot \ddot{\bar{u}}_{s 2} *+\dot{\bar{i}}_{r 2}-c_{3} \cdot k_{5} \cdot \dot{e}_{5}-k_{6} \cdot e_{6}\right]$

\section{Simulation Results}

The PV model, DC-DC and DC-AC converters and the backstepping controllers are implemented in Matlab/Simulink as illustrated in Fig. 6. In the study, RSM-60 has been selected as PV source. The PV generator is chosen to deliver maximum $1 \mathrm{~kW}$ by 
connecting 16 module parallely. The specification of the system and PV module are respectively summarized in the Table 1 and 2.

A Matlab/Simulink simulation of the PV system, the MPPT algorithm and the backstepping controller been carried out using the parameters in Table 2 .

The studied PV system is evaluated on two aspects: The first one to extract the maximum power according to solar radiation and temperature change. The second one is the ability to regulate the outputs DC voltage and AC voltage to a references.

Figure $7 \mathrm{a}$ and $\mathrm{b}$ shows the irradiation and temperature change respectively.

Figure 7c shows the PV power properly following its maximum value according to irradiation and temperature change.

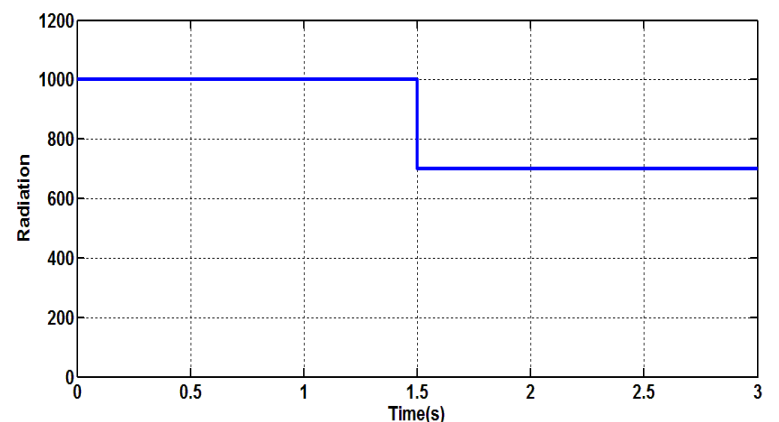

(a)

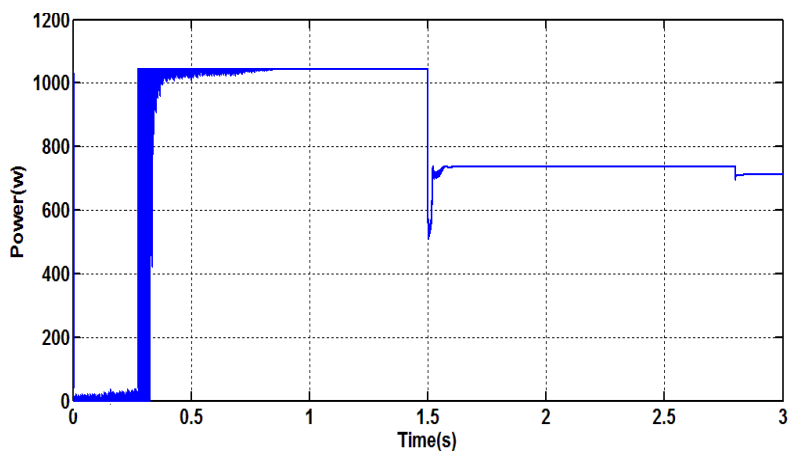

(c)
Figure $7 \mathrm{~d}$ illustrates the output $\mathrm{DC}$ voltage properly following its reference value ( $12 \mathrm{~V}$ or $24 \mathrm{~V})$.

Table 1. Main characteristics of the PV generation system

\begin{tabular}{llll}
\hline $\begin{array}{l}\text { Maximum } \\
\text { power }\end{array}$ & $\begin{array}{l}\text { Output } \\
\text { voltage at } \mathrm{P}_{\max }\end{array}$ & $\begin{array}{l}\text { Open-circuit } \\
\text { voltage }\end{array}$ & $\begin{array}{l}\text { Short } \\
\text { current circuit }\end{array}$ \\
\hline $60 \mathrm{w}$ & $16 \mathrm{v}$ & $21.5 \mathrm{v}$ & $3.8 \mathrm{~A}$ \\
\hline
\end{tabular}

Table 2. Control parameters used in simulation

\begin{tabular}{lcl}
\hline Parameters & Value & Unit \\
\hline $\mathrm{c}_{1}$ & 220 & $\mu \mathrm{F}$ \\
$\mathrm{c}_{2}$ & 470 & $\mu \mathrm{F}$ \\
$\mathrm{c}_{3}$ & 22 & $\mu \mathrm{F}$ \\
$\mathrm{L}_{1}$ & 2 & $\mathrm{mH}$ \\
$\mathrm{L}_{2}$ & 2 & $\mathrm{mH}$ \\
$\mathrm{L}_{3}$ & 1 & $\mathrm{mH}$ \\
\hline
\end{tabular}

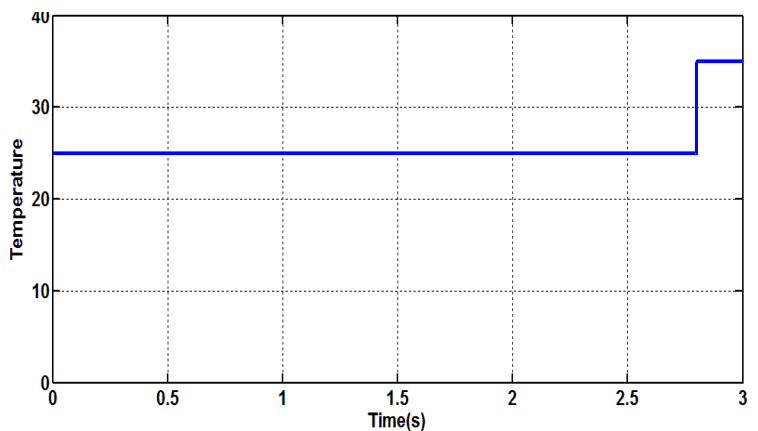

(b)

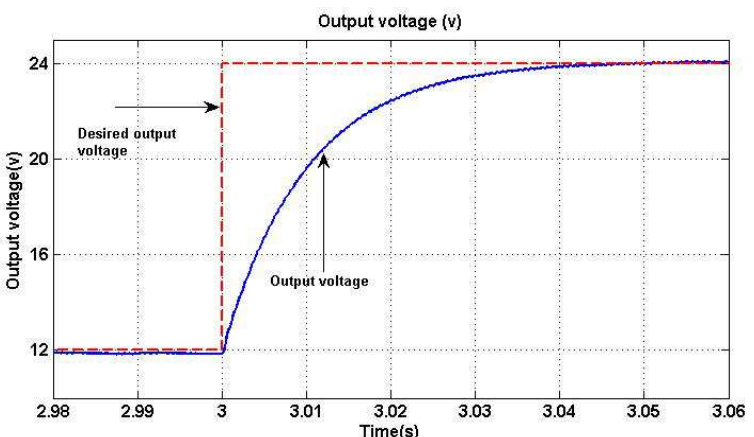

(d)

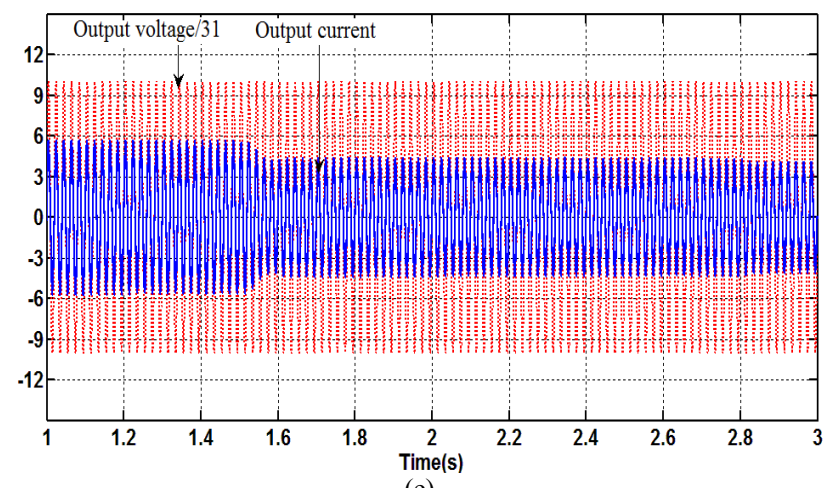

(e)

Fig. 7. (a) Radiation, (b) Temperature, (c) PV Power, (d) Output voltage $u_{\mathrm{s} 1}$, (e) Output voltage $\mathrm{u}_{\mathrm{s} 2}$ 
Figure $7 \mathrm{e}$ shows that the output $\mathrm{AC}$ voltage and output current. This figure illustrates the output voltage well achieved to its reference $(220 \mathrm{~V} / 50 \mathrm{~Hz})$.

These simulations show the effectiveness of the combination of backstepping controller approach and MPPT to search the maximum power and ability to adjustment of outputs voltages without influence of climate change.

\section{Conclusion}

A backstepping control strategy has been developed in the PV generating system. The first objective is to ensure operation at maximum power of solar panels. The second objective is to regulate the output voltage of the continuous and alternative load. The simulations results have shown that the objectives are achieved and that the PV system is stable and better efficiency against climate change.

\section{Acknowledgment}

I thank all those who contribute near and far to the development of this work whether with ideas or the discussions.

\section{Funding Information}

There is no funding for this work. this work is developed in a personal capacity

\section{Ethics}

This scientific research work is developped in the direction of improving the energy production for a global population increasingly growing and also to respond to the global economic demand. This work is considered part of a thesis on the ability of $\mathrm{H}$. Abouobaida and will be published shortly.

\section{References}

Barth, C. and R.C.N. Pilawa-Podgurski, 2013. Dithering digital ripple correlation control for photovoltaic maximum power point tracking. Proceedings of the IEEE Power and Energy Conference at Illinois, Feb. 22-23, IEEE Xplore Press, Champaign, IL., pp: 36-41. DOI: 10.1109/PECI.2013.6506031

Casadei, D., G. Grandi and C. Rossi, 2006. Single-phase single-stage photovoltaic generation system based on a ripple correlation control maximum power point tracking. IEEE Trans. Energy Convers., 21: 562-568. DOI: 10.1109/TEC.2005.853784

Lalouni, S. and D. Rekioua, 2009. Modeling and simulation of a photovoltaic system using fuzzy logic controller. Proceedings of the 2 nd International Conference on Developments in eSystems Engineering, Dec. 14-16, IEEE Xplore Press, Abu Dhabi, pp: 23-28. DOI: 10.1109/DeSE.2009.17

Liu, S., H. Liu and Y. Zhao, 2013. A solar maximum power point tracking algorithm based on discretetime ripple correlation control. Trans. Chinese Society Agric. Eng., 29: 130-137.

Rekioua, D. and E. Matagne, 2012. Optimization of Photovoltaic Power Systems: Modelization, Simulation and Control. 1st Edn., Springer Science and Business Media, ISBN-10: 1447123484, pp: 283. 